\title{
Disease-specific mortality among stage I-III colorectal cancer patients with diabetes: a large population-based analysis
}

\author{
L. V. van de Poll-Franse • H. R. Haak • \\ J. W. W. Coebergh • M. L. G. Janssen-Heijnen • \\ V. E. P. P. Lemmens
}

Received: 26 October 2011 / Accepted: 14 March 2012 /Published online: 24 April 2012

(C) The Author(s) 2012. This article is published with open access at Springerlink.com

\begin{abstract}
Aims/hypothesis The aim of our study was to investigate overall and disease-specific mortality of colorectal cancer patients with diabetes.

Methods In this population-based study, we included all colorectal cancer patients, newly diagnosed with stage I-III cancer, between 1997 and 2007 in the registration area of the Eindhoven Cancer Registry. Stage of cancer, cancer treatment and comorbidities were actively collected by reviewing hospital medical records. Data on patients with and without diabetes were linked to Statistics Netherlands to
\end{abstract}

L. V. van de Poll-Franse $(\bowtie) \cdot$ J. W. W. Coebergh •

M. L. G. Janssen-Heijnen • V. E. P. P. Lemmens

Comprehensive Cancer Centre South

(CCCS)/Eindhoven Cancer Registry,

PO Box 231, 5600 AE Eindhoven, the Netherlands

e-mail: L.vd.poll@ikz.nl

L. V. van de Poll-Franse

e-mail: 1.vd.poll@uvt.nl

L. V. van de Poll-Franse

CoRPS - Center of Research on Psychology in Somatic Diseases,

Department of Medical Psychology, Tilburg University,

Tilburg, the Netherlands

\section{H. R. Haak}

Department of Internal Medicine, Maxima Medical Centre,

Eindhoven, the Netherlands

J. W. W. Coebergh • V. E. P. P. Lemmens

Department of Public Health, Erasmus Medical Centre,

Rotterdam, the Netherlands

M. L. G. Janssen-Heijnen

Department of Clinical Epidemiology, Viecuri Medical Centre,

Venlo, the Netherlands assess vitality, date of death and underlying cause of death. Follow-up of all patients was completed until 1 January 2009.

Results We included 6,974 patients with colon cancer and 3,888 patients with rectal cancer, of whom $820(12 \%)$ and $404(10 \%)$, respectively, had diabetes at the time of cancer diagnosis. During follow-up, death occurred in $611(50 \%)$ of 1,224 cancer patients with diabetes and 3,817 (40\%) of 9,638 cancer patients without diabetes. Multivariate Cox regression analyses, adjusted for age, sex, socioeconomic status, stage, lymph nodes examined, adjuvant therapy and year of diagnosis, showed that overall mortality was significantly higher for colon (HR 1.12, 95\% CI 1.01, 1.25) and rectal (HR 1.21, 95\% CI 1.03, 1.41) cancer patients with diabetes than for those without. Disease-specific mortality was only significantly increased for rectal cancer patients (HR 1.30, 95\% CI 1.06, 1.60).

Conclusions/interpretation Diabetes at the time of rectal cancer diagnosis was independently associated with an increased risk of colorectal cancer mortality compared with no diabetes, suggesting a specific interaction between diabetes and rectal cancer. Future in-depth studies including detailed diabetes- and cancer-related variables should elucidate pathways.

Keywords Colorectal cancer · Diabetes · Disease-specific mortality $\cdot$ Population-based
Abbreviations
CVA Cerebrovascular accident
CVD Cardiovascular disease
ECR Eindhoven Cancer Registry
SES Socioeconomic status
UCOD Underlying cause of death 


\section{Introduction}

The number of older people with cancer is increasing considerably, resulting in a growing proportion of patients who present with multiple coexisting medical conditions [1]. Worldwide, a strongly increasing prevalence of diabetes has been observed [2-4]. Breast, colorectal, endometrial, pancreatic and kidney cancer are more prevalent in patients with diabetes than in those without diabetes. Furthermore, the prevalence of diabetes in these patients is also higher than in other cancer patients or patients without cancer [5]. It is estimated that the number of newly diagnosed cancer patients with diabetes in the Netherlands will double from 5,500 in 2000 to 10,400 in 2015 [6].

Since 1993, the Eindhoven Cancer Registry (ECR) has registered the prevalence of comorbid conditions at the time of cancer diagnosis. About $60 \%$ of all newly diagnosed cancer patients older than 65 years had at least one other serious disease, of which diabetes was one of the most common (16\%) [1]. In a population-based analysis of all 58,498 cancer patients diagnosed between 1995 and 2002 in the southern Netherlands, we showed that patients with preexisting diabetes had a significantly increased overall mortality [5]. This result was confirmed by a meta-analysis that included our paper and 22 other papers [7], and a review that focused on colorectal cancer [8].

In 2010, the American Diabetes Association and American Cancer Society reviewed the state of science concerning diabetes and cancer $[9,10]$. One of the key issues was a better understanding of whether diabetes influences cancer prognosis above and beyond the prognosis conferred by each disease state independently. Today, only a few studies have evaluated disease-specific mortality among cancer patients with diabetes. Poorly controlled, pre-existing diabetes mellitus has been associated with an increased risk of death attributable to colorectal cancer [11]. Similarly, hyperinsulinaemia and five other previously established components of metabolic syndrome were shown to be prospective risk factors for deaths that can be ascribed to prostate cancer [12]. Furthermore, an increased risk was also observed for endometrial cancer mortality among women with diabetes [13]. Recently, the large prospective Cancer Prevention Study-II Nutrition Cohort showed increased colorectal cancer mortality among patients with colorectal cancer and diabetes [14]. However, other studies found no association between diabetes- and cancer-specific mortality [15-18]. These conflicting results may be explained by small numbers of diabetes patients, a limited number of deaths, and/or no information about cancer treatment [11-13, 15-18].

The aim of our study was to investigate the overall and disease-specific mortality of colorectal cancer patients with diabetes, taking into account differences in age, comorbidity (especially cardiovascular diseases [CVDs]), stage and treatment according to diabetes status. In view of the variable associations between diabetes and cancer risk at specific sites $[9,10,19]$, we analysed our data for colon and rectal cancer separately.

\section{Methods}

Patients and methods The ECR records data on all patients newly diagnosed with cancer in the southern part of the Netherlands, an area with 2.3 million inhabitants, ten general hospitals and two large radiotherapy institutes. Trained registration clerks actively collect data on diagnosis and staging (tumour, nodes, metastasis [TNM]) and detailed information about initial treatment from hospital medical records. Registration takes place 6-12 months after diagnosis. By means of an independent case ascertainment method, the completeness of the registration has been estimated to exceed $95 \%$ [20]. The medical record is generally regarded as the most complete source of information on the patient's past and current health status [21]. Since 1993, serious comorbidity with prognostic impact has been recorded for all patients. A slightly modified version of the widely used Charlson comorbidity index [22] is used [1]. Comorbidity was defined as life-shortening diseases that were present at the time of cancer diagnosis. Drug use, often recorded from general practitioners' letters, served as an indicator of active disease. Diabetes mellitus included both type I and type II disease and was registered as a dichotomous variable (yes/no), as were all other concomitant conditions. CVD included myocardial infarction, cardiac insufficiency, angina pectoris, coronary artery bypass graft, peripheral arterial disease, and cerebrovascular diseases. Comorbidity data since 1995 are considered accurate, i.e. complete and correct.

We included all colorectal cancer (International Classification of Diseases for Oncology codes C18.0-C20.9) patients, newly diagnosed with stage I-III cancer, between 1997 and 2007. We excluded patients for whom the site of the primary tumour within the colorectum was not known (1.5\% of total). Oncological treatment was defined as surgery, radiotherapy and/or chemotherapy. Surgery did not comprise diagnostic operations. Data on patients with colorectal cancer with or without diabetes from the populationbased ECR were linked to mortality data from the database of Statistics Netherlands.

Statistics Netherlands registers vital status, including, if applicable, date of death of a person as recorded by the municipal population registries. Statistics Netherlands also documents the primary underlying cause of death (UCOD) as recorded by the attending physician. This is performed according to the World Health Organization UCOD definition 
'the disease or injury which initiated the chain of morbid events leading directly to death, or the circumstances of the accident or violence which produced the fatal injury' and in accordance with the rules of the International Classification of Diseases-tenth revision. Follow-up of all patients was completed until 1 January 2009.

Postal codes of residential areas are used to establish the socioeconomic status (SES) of diagnosed cancer patients, also provided by Statistics Netherlands [23]. At the sixposition level of postal code, data on household income and economical value of the house are available from fiscal data. This information was transformed into four categories: low, medium and high SES and patients who were institutionalised (such as in a nursing home). ECR and Statistics Netherlands data were linked by use of postal codes and dates of birth. If patients could not be found in Statistics Netherlands with this strategy, date of death was also provided by the ECR to track the patient in Statistics Netherlands files.

Statistical analysis The SAS computer package (version 9.1 for Windows; SAS Institute, Cary, NC, USA) was used for all statistical analyses. All analyses were performed separately for colon and rectal cancer patients. Differences in patient, tumour and treatment characteristics according to diabetes status were tested by $\chi^{2}$ analysis or $t$ test, as appropriate. Non-parametric equivalents were applied when normality and homogeneity assumptions were violated. Survival analysis was carried out using the life table method to evaluate the prognosis after diagnosis for cancer patients with or without diabetes. Survival time was defined as the time from diagnosis to death or 1 January 2009 for the patients who were still alive. Five-year cumulative survival probability and its standard error were calculated for those with or without diabetes and compared by means of the logrank test.

The independent prognostic effect of diabetes on overall mortality and colorectal cancer-specific mortality was estimated using Cox proportional hazards regression models. The proportional hazard assumption of diabetes was evaluated by estimating Kaplan-Meier curves. The effect of diabetes over time satisfied the assumption of proportionality as the graphs of the $\log (\log [$ survival]) versus $\log$ of survival time exhibited parallel lines for the groups of diabetic and non-diabetic patients. Confounding variables included for adjustment in the Cox proportional hazards regression models were determined a priori [24]: age at diagnosis, sex, stage, number of examined lymph nodes $(\geq 10)$, adjuvant therapy (chemotherapy or radiotherapy), SES, year of diagnosis, hypertension, CVD, cerebrovascular disease, previous cancer and lung disease. Effect modification between diabetes and all potential confounding variables was assessed by adding interaction terms diabetes $\times$ confounding variable in the multivariate Cox proportional hazards regression models. HRs with $95 \%$ CIs and $p$ values are reported.

\section{Results}

Between 1997 and 2007, 15,655 colorectal cancer patients were diagnosed in the ECR area (Fig. 1). Of these, 15,449 (99\%) were successfully linked with Death Statistics Netherlands. After exclusion of patients with stage IV disease or missing information on stage, we included 6,974 colon cancer and 3,888 rectal cancer patients with stage I-III disease, of whom $820(12 \%)$ and $404(10 \%)$, respectively, had diabetes at cancer diagnosis. Colon cancer patients with diabetes were on average 4 years older than their counterparts without diabetes, had a lower SES, more additional comorbid conditions and less often received adjuvant systemic therapy (Table 1). Colon cancer patients with diabetes had more often been diagnosed with a previous cancer, hypertension, CVD, cerebrovascular accident (CVA) and lung disease than those without diabetes. There were no differences in sex, stage, grade and number of lymph nodes examined. Although not statistically significant, colon cancer patients with diabetes appeared to be diagnosed more often in more recent periods than those without diabetes. Rectal cancer patients with diabetes were on average 5 years older than their counterparts without diabetes, and had a lower SES and more additional comorbid conditions (Table 2). Rectal cancer patients with diabetes also had been diagnosed more often with a previous cancer, hypertension, CVD, CVA and lung disease. There were no differences in the presence of diabetes with respect to sex, stage, grade, number of examined lymph nodes, and receipt of adjuvant therapy or period of diagnosis.

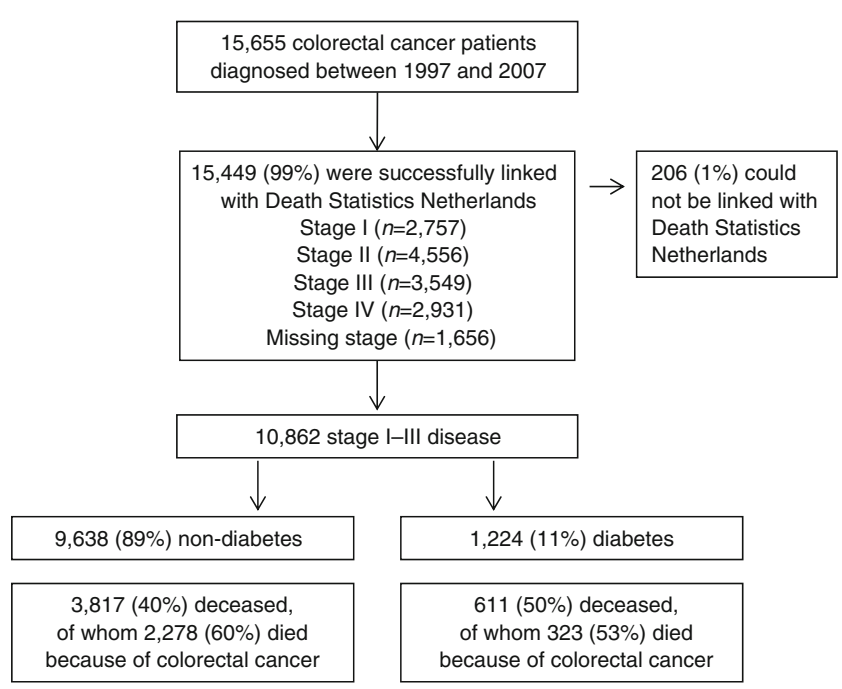

Fig. 1 Flow chart of patient selection 
Table 1 Characteristics of patients with colon cancer stage I-III according to diabetes status

\begin{tabular}{|c|c|c|c|}
\hline Characteristic & $\begin{array}{l}\text { Diabetes } \\
(n=820)\end{array}$ & $\begin{array}{l}\text { No diabetes } \\
(n=6,154)\end{array}$ & $p$ value \\
\hline Male & $376(46)$ & $3,119(51)$ & 0.0094 \\
\hline Age (years) & $73.0 \pm 8.8$ & $68.8 \pm 11.1$ & $<0.0001$ \\
\hline \multicolumn{4}{|l|}{ Age } \\
\hline $0-59$ & $66(8)$ & $1,218(20)$ & \\
\hline $60-69$ & $191(23)$ & $1,707(28)$ & \\
\hline $70-79$ & $354(43)$ & $2,201(36)$ & \\
\hline $80+$ & $209(25)$ & $1,028(17)$ & $<0.0001$ \\
\hline \multicolumn{4}{|l|}{ Stage } \\
\hline I & $150(18)$ & $1,221(20)$ & \\
\hline II & $409(50)$ & $2,849(46)$ & \\
\hline III & $261(32)$ & $2,084(34)$ & 0.15 \\
\hline \multicolumn{4}{|l|}{ Examined lymph nodes } \\
\hline Median (25-75\%) & $7(3-11)$ & $7(4-11)$ & \\
\hline$\geq 10$ & $197(32)$ & $1,544(33)$ & 0.48 \\
\hline \multicolumn{4}{|l|}{ Therapy } \\
\hline Surgery alone & $677(83)$ & $4,715(77)$ & \\
\hline Surgery and systemic & $118(14)$ & $1,257(20)$ & \\
\hline Other/unknown & $25(3)$ & $182(3)$ & 0.0008 \\
\hline \multicolumn{4}{|l|}{ SES } \\
\hline Low & $291(36)$ & $1,610(27)$ & \\
\hline Medium & $276(34)$ & $2,305(38)$ & \\
\hline High & $174(22)$ & $1,819(30)$ & \\
\hline Institutionalised & $68(8)$ & $296(5)$ & $<0.0001$ \\
\hline \multicolumn{4}{|c|}{ Comorbidity, excluding diabetes } \\
\hline 0 & $163(20)$ & $2,098(34)$ & \\
\hline 1 & $285(35)$ & $1,946(32)$ & \\
\hline 2 or more & $372(45)$ & $1,450(24)$ & \\
\hline Missing & $0(-)$ & $660(11)$ & $<0.0001$ \\
\hline \multicolumn{4}{|l|}{ Type of comorbidity } \\
\hline Previous cancer & $135(16)$ & $822(13)$ & 0.015 \\
\hline Hypertension & $365(45)$ & $1,242(20)$ & $<0.0001$ \\
\hline CVD & $344(42)$ & $1,441(23)$ & $<0.0001$ \\
\hline CVA & $80(10)$ & $239(4)$ & $<0.0001$ \\
\hline Lung diseases & $101(12)$ & $557(9)$ & 0.003 \\
\hline \multicolumn{4}{|l|}{ Period of cancer diagnosis } \\
\hline 1997-1999 & $176(21)$ & $1,401(23)$ & \\
\hline $2000-2002$ & $200(24)$ & $1,721(28)$ & \\
\hline $2003-2005$ & $255(31)$ & $1,746(28)$ & \\
\hline 2006-2007 & $189(23)$ & $1,286(21)$ & 0.061 \\
\hline
\end{tabular}

Unless otherwise stated, values are $n(\%)$ or mean $\pm \mathrm{SD}$

Of all 10,862 patients diagnosed in the period 1997 $2007,4,428(41 \%)$ deaths were registered up to the follow-up date 1 January 2009 (Table 3). Evaluation of UCOD showed that colon cancer patients most often died of colon cancer $(52 \%$ of those without diabetes vs $44 \%$ with diabetes), whereas in $2 \%$ of the patients, rectal cancer was
Table 2 Characteristics of patients with rectal cancer stage I-III according to diabetes status

\begin{tabular}{|c|c|c|c|}
\hline Characteristic & $\begin{array}{l}\text { Diabetes } \\
(n=404)\end{array}$ & $\begin{array}{l}\text { No diabetes } \\
(n=3,484)\end{array}$ & $p$ value \\
\hline Male & $230(57)$ & $2,081(57)$ & 0.28 \\
\hline Age (years) & $71.2 \pm 8.4$ & $66.1 \pm 11.2$ & $<0.0001$ \\
\hline \multicolumn{4}{|l|}{ Age } \\
\hline $0-59$ & $42(10)$ & $964(28)$ & \\
\hline $60-69$ & $121(30)$ & $1,119(32)$ & \\
\hline $70-79$ & $173(43)$ & $1,004(29)$ & \\
\hline $80+$ & $68(17)$ & $397(11)$ & $<0.0001$ \\
\hline \multicolumn{4}{|l|}{ Stage } \\
\hline I & $133(33)$ & $1,253(36)$ & \\
\hline II & $142(35)$ & $1,156(33)$ & \\
\hline III & $129(32)$ & $1,075(31)$ & 0.47 \\
\hline \multicolumn{4}{|l|}{ Examined lymph nodes } \\
\hline Median (25-75\%) & $5(2-8)$ & $5(2-9)$ & \\
\hline$\geq 10$ & $68(21)$ & $529(20)$ & 0.52 \\
\hline \multicolumn{4}{|l|}{ Therapy } \\
\hline Surgery alone & $152(38)$ & $1,156(33)$ & \\
\hline Surgery + radiotherapy & $183(45)$ & $1,647(47)$ & \\
\hline Surgery + radiotherapy + systemic & $40(10)$ & $408(12)$ & \\
\hline Surgery + systemic & $14(3)$ & $146(4)$ & \\
\hline Other/unknown & $15(3)$ & $127(4)$ & 0.23 \\
\hline \multicolumn{4}{|l|}{ SES } \\
\hline Low & $134(34)$ & $794(23)$ & \\
\hline Medium & $152(38)$ & $1,364(40)$ & \\
\hline High & $88(22)$ & $1,111(33)$ & \\
\hline Institutionalised & $26(7)$ & $139(4)$ & $<0.0001$ \\
\hline \multicolumn{4}{|l|}{ Comorbidity, excluding diabetes } \\
\hline 0 & 79 (19) & $1,436(41)$ & \\
\hline 1 & $152(38)$ & $1,060(30)$ & \\
\hline 2 or more & $173(43)$ & $634(18)$ & \\
\hline Missing & $0(-)$ & $660(11)$ & $<0.0001$ \\
\hline \multicolumn{4}{|l|}{ Type of comorbidity } \\
\hline Previous cancer & $67(17)$ & $344(10)$ & $<0.0001$ \\
\hline Hypertension & $195(48)$ & $669(19)$ & $<0.0001$ \\
\hline CVD & $154(38)$ & $650(19)$ & $<0.0001$ \\
\hline CVA & $34(8)$ & $105(3)$ & $<0.0001$ \\
\hline Lung diseases & $46(11)$ & $294(8)$ & 0.047 \\
\hline \multicolumn{4}{|l|}{ Period of cancer diagnosis } \\
\hline 1997-1999 & $79(20)$ & $781(22)$ & \\
\hline $2000-2002$ & $100(25)$ & $927(27)$ & \\
\hline $2003-2005$ & $134(33)$ & $1,065(31)$ & \\
\hline $2006-2007$ & $91(23)$ & $711(20)$ & 0.33 \\
\hline
\end{tabular}

Unless otherwise stated, values are $n(\%)$ or mean \pm SD

registered as the UCOD. Of the patients diagnosed with rectal cancer, $23 \%$ had colon cancer registered as their UCOD. As misclassification of cause of death may have played a role here, we decided to investigate colorectal 
Table 3 Number and percentages of patients, deaths, causes of death and survival according to cancer subsite and diabetes status

Values are $n(\%)$ or $\%$

CRC, colorectal cancer; NOS, not otherwise specified

${ }^{a}$ Colon cancer as underlying cause of death

${ }^{\mathrm{b}}$ Rectal cancer as underlying cause of death

\begin{tabular}{|c|c|c|c|c|}
\hline \multirow[t]{2}{*}{ Characteristic } & \multicolumn{2}{|l|}{ Colon } & \multicolumn{2}{|l|}{ Rectal } \\
\hline & Diabetes & No diabetes & Diabetes & No diabetes \\
\hline Number of patients & 820 & 6,154 & 404 & 3,484 \\
\hline Number of deaths & $417(51)$ & $2,525(41)$ & $194(48)$ & $1,292(37)$ \\
\hline \multicolumn{5}{|l|}{ UCOD } \\
\hline $\mathrm{CRC}$ & $194(47)$ & $1,376(55)$ & $110(57)$ & $759(59)$ \\
\hline Colon cancer ${ }^{\mathrm{a}}$ & $184(44)$ & $1,313(52)$ & $45(23)$ & $301(23)$ \\
\hline Rectal cancer ${ }^{\mathrm{b}}$ & $10(2)$ & $63(2)$ & $65(34)$ & $458(35)$ \\
\hline NOS digestive cancer & $24(6)$ & $159(6)$ & $8(4)$ & $84(7)$ \\
\hline Other cancers & $42(10)$ & $292(12)$ & $13(7)$ & $126(10)$ \\
\hline Diabetes & $20(5)$ & $7(0.3)$ & $5(3)$ & $3(0.2)$ \\
\hline Circulatory system & $75(18)$ & $332(13)$ & $36(19)$ & $138(11)$ \\
\hline Respiratory disease & $21(5)$ & $98(4)$ & $7(4)$ & $58(4)$ \\
\hline External causes & $4(1)$ & $26(1)$ & $1(0.5)$ & $16(1)$ \\
\hline Infections & $3(0.7)$ & $23(1)$ & $1(0.5)$ & $17(1)$ \\
\hline Other & $34(8)$ & $212(8)$ & $13(7)$ & $91(7)$ \\
\hline \multicolumn{5}{|l|}{ Survival } \\
\hline 5-year overall & 51 & 60 & 50 & 64 \\
\hline 10 -year overall & 22 & 22 & 31 & 48 \\
\hline 5-year CRC-specific & 73 & 75 & 67 & 75 \\
\hline 10-year CRC-specific & 61 & 69 & 60 & 70 \\
\hline
\end{tabular}

cancer death as one group in patients diagnosed with colon or rectal cancer. Therefore, throughout this paper, diseasespecific survival or mortality always includes both colon and rectal cancer as the primary cause of death or underlying complication. Cancer patients with diabetes more often had diabetes registered as UCOD (4\%) or diseases of the circulatory system (18\%) compared with cancer patients without diabetes ( $0 \%$ and $12 \%$, respectively).

Compared with patients without diabetes, 5-year overall survival was 9\% lower among colon cancer patients and $14 \%$ lower among rectal cancer patients with diabetes. The 10 -year overall survival rates were $22 \%$ and $17 \%$ lower, respectively. Differences in cancer-specific 5- and 10-year survival were somewhat smaller (2-9\%).

Unadjusted analyses revealed that the overall survival of cancer patients without diabetes was significantly higher than survival of those with diabetes (Figs 2, 3, 4 and 5). For colon and rectal cancer, the observed unadjusted HR for the effect of diabetes on overall mortality was 1.42 (95\% CI $1.28,1.57)$ and $1.58(95 \%$ CI $1.36,1.84)$, respectively. Multivariate Cox proportional hazards regression analyses, with adjustment for differences in age, sex, SES, stage, number of lymph nodes examined, receipt of adjuvant therapy and period of diagnosis, showed that overall mortality remained significantly higher for colon (HR 1.22, 95\% CI $1.09,1.34$ ) and rectal (HR 1.29, 95\% CI 1.11, 1.50) cancer patients with diabetes (data not shown). Bivariate analyses showed that age, sex and therapy were the strongest confounding variables that resulted in a dilution of the effect of diabetes on overall mortality. Further adjustment for hypertension, CVD, cerebrovascular disease, previous cancer and lung disease lowered the HR for overall mortality even further in colon (HR 1.12, 95\% CI 1.01, 1.25; Table 4) and rectal (HR 1.21, 95\% CI 1.03, 1.41; Table 5) cancer patients with diabetes. CVD was the strongest additional confounding factor in this step, although diabetes remained an independent predictor of overall mortality in colon and rectal cancer patients.

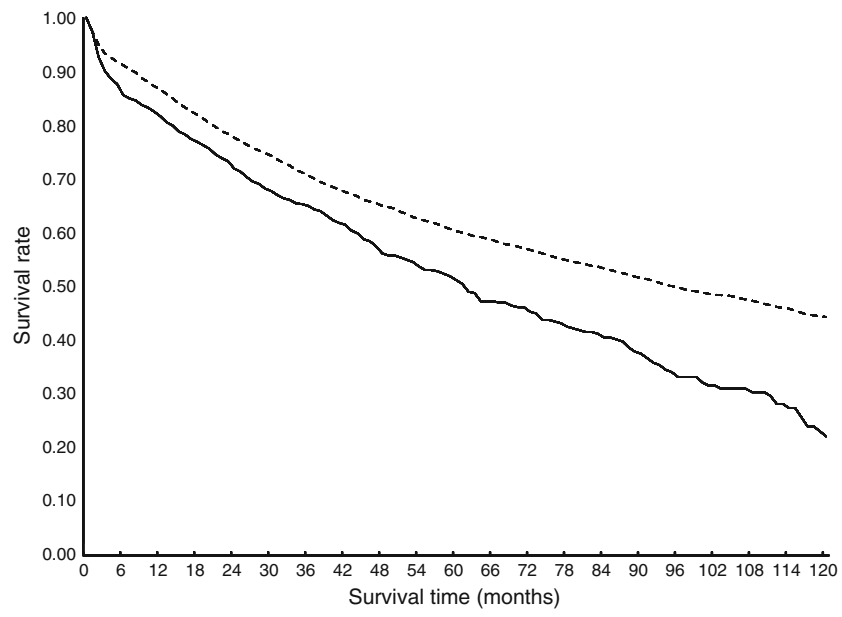

Fig. 2 Overall survival for stage I-III colon cancer patients with (solid line) or without (dashed line) diabetes 


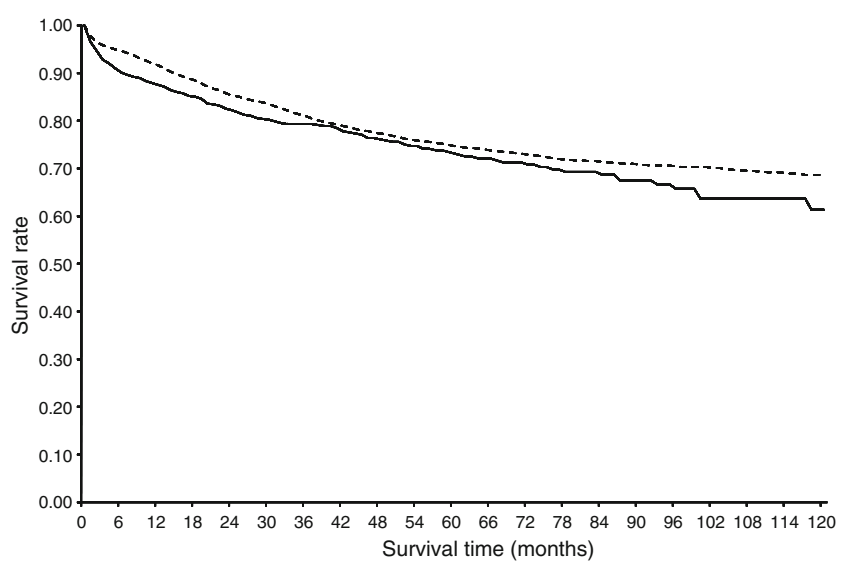

Fig. 3 Colorectal cancer-specific survival for stage I-III colon cancer patients with (solid line) or without (dashed line) diabetes

Cancer-specific mortality was also increased among colorectal cancer patients with diabetes. Unadjusted analyses showed a small increased risk of cancer-specific mortality in colon cancer patients with diabetes compared with those without diabetes (HR 1.17, 95\% CI 1.01, 1.36; data not shown). However, after adjustment for confounding variables, this increased risk disappeared (HR 1.05, 95\% CI 0.90, 1.23; Table 4). Bivariate analyses showed that age, sex and receipt of adjuvant therapy were the strongest confounding variables, whereas addition of having had a previous cancer or other comorbidities to the multivariate model did not significantly change the observed HR. We also observed a statistically significant interaction between diabetes and sex for patients with colon cancer. Women with colon cancer and diabetes had an increased risk of colon cancer mortality, whereas men did not. However, after adjustment for age, this finding disappeared.

Cancer-specific mortality was significantly increased among rectal cancer patients with diabetes compared with those without (HR 1.48; 95\% CI 1.21, 1.81; data not shown). After adjustment, cancer-specific mortality remained

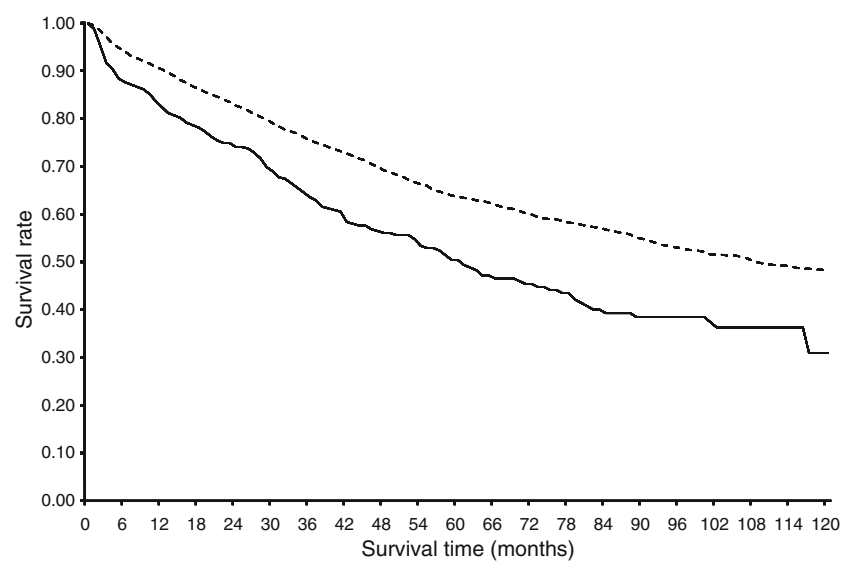

Fig. 4 Overall survival for stage I-III rectal cancer patients with (solid line) or without (dashed line) diabetes

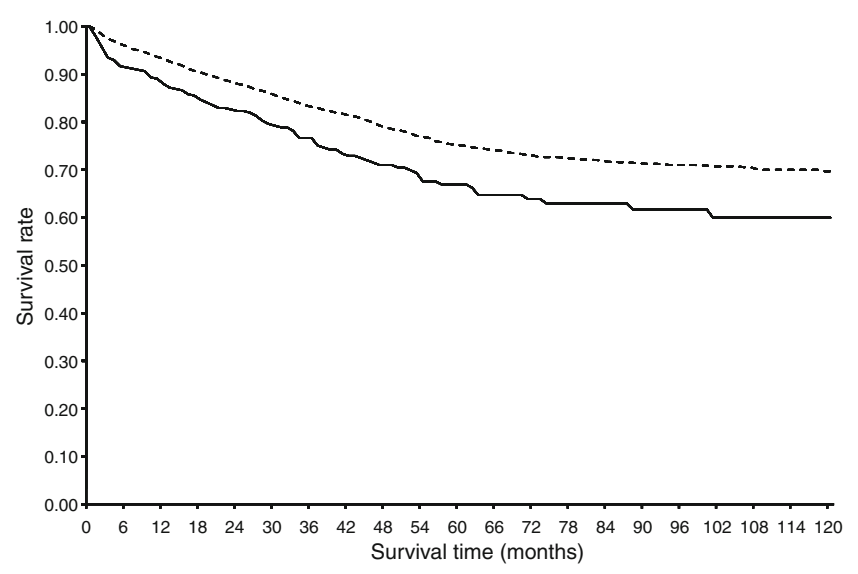

Fig. 5 Colorectal cancer-specific survival for stage I-III rectal cancer patients with (solid line) or without (dashed line) diabetes

Table 4 Multivariate Cox regression analyses of the effect of diabetes on overall and colorectal cancer-specific mortality in stage I-III colon cancer

\begin{tabular}{|c|c|c|}
\hline Characteristic & Overall mortality & $\begin{array}{l}\text { Colorectal cancer } \\
\text { mortality }\end{array}$ \\
\hline Diabetes (vs no) & $1.12(1.01,1.25)^{*}$ & $1.05(0.90,1.23)$ \\
\hline Male sex (vs female sex) & $1.22(1.13,1.31)^{* *}$ & $1.12(1.01,1.24)^{*}$ \\
\hline Age (per 1 year increase) & $1.04(1.04,1.05)^{* *}$ & $1.02(1.01,1.03)^{* *}$ \\
\hline \multicolumn{3}{|l|}{ Period of cancer diagnosis } \\
\hline 1997-1999 & 1 & 1 \\
\hline $2000-2002$ & $0.99(0.89,1.10)$ & $0.89(0.77,1.03)$ \\
\hline $2003-2005$ & $0.93(0.82,1.04)$ & $0.91(0.77,1.06)$ \\
\hline 2006-2007 & $0.80(0.68,0.94)^{* *}$ & $0.86(0.70,1.06)$ \\
\hline \multicolumn{3}{|l|}{ Stage } \\
\hline I & 1 & 1 \\
\hline II & $1.64(1.46,1.83)^{* *}$ & $2.60(2.14,3.17)^{* *}$ \\
\hline III & $3.17(2.80,3.60)^{* *}$ & $6.83(5.56,8.39)^{* *}$ \\
\hline $\begin{array}{l}\text { Examined lymph nodes } \geq 10 \\
\quad \text { (vs no) }\end{array}$ & $0.69(0.62,0.77)^{* *}$ & $0.62(0.53,0.71)^{* *}$ \\
\hline Systemic therapy (vs no) & $0.68(0.61,0.77)^{* *}$ & $0.69(0.59,0.80)^{* *}$ \\
\hline \multicolumn{3}{|l|}{ SES } \\
\hline Low & 1 & 1 \\
\hline Medium & $0.94(0.86,1.03)$ & $0.98(0.87,1.11)$ \\
\hline High & $0.87(0.79,0.96)^{* *}$ & $0.91(0.70,1.05)$ \\
\hline Institutionalised & $1.09(0.94,1.27)$ & $0.96(0.77,1.20)$ \\
\hline Hypertension (vs no) & $0.97(0.89,1.06)$ & $0.89(0.79,1.01)$ \\
\hline CVD (vs no) & $1.33(1.23,1.45)^{* *}$ & $1.12(0.99,1.26)$ \\
\hline CVA (vs no) & $1.32(1.14,1.53)^{* *}$ & $1.21(0.97,1.50)$ \\
\hline Previous cancer (vs no) & $1.32(1.20,1.46)^{* *}$ & $1.26(1.10,1.45)^{* *}$ \\
\hline Lung disease (vs no) & $1.16(1.04,1.30)^{* *}$ & $1.09(0.93,1.29)$ \\
\hline
\end{tabular}


Table 5 Multivariate Cox regression analyses of the effect of diabetes on overall and colorectal cancer-specific mortality in stage I-III rectal cancer

\begin{tabular}{|c|c|c|}
\hline Characteristic & Overall mortality & $\begin{array}{l}\text { Colorectal cancer } \\
\text { mortality }\end{array}$ \\
\hline Diabetes (vs no) & $1.21(1.03,1.41)^{*}$ & $1.30(1.06,1.60)^{*}$ \\
\hline Male sex (vs female sex) & $1.16(1.04,1.29)^{* *}$ & $1.14(0.99,1.31)$ \\
\hline Age (per 1 year increase) & $1.05(1.04,1.05)^{* *}$ & $1.03(1.03,1.04)^{* *}$ \\
\hline \multicolumn{3}{|l|}{ Period of cancer diagnosis } \\
\hline 1997-1999 & 1 & 1 \\
\hline $2000-2002$ & $1.11(0.96,1.29)$ & $0.92(0.76,1.11)$ \\
\hline 2003-2005 & $0.82(0.69,0.97)^{*}$ & $0.66(0.53,0.82)^{* *}$ \\
\hline 2006-2007 & $0.76(0.61,0.97)^{*}$ & $0.60(0.45,0.81)^{* *}$ \\
\hline \multicolumn{3}{|l|}{ Stage } \\
\hline I & 1 & 1 \\
\hline II & $1.80(1.58,2.06)^{* *}$ & $3.03(2.46,3.73)^{* *}$ \\
\hline III & $2.66(2.33,3.05)^{* *}$ & $5.02(4.07,6.18)^{* *}$ \\
\hline $\begin{array}{l}\text { Examined lymph nodes } \geq 10 \\
\quad \text { (vs no) }\end{array}$ & $0.84(0.71,1.00)^{*}$ & $0.79(0.63,0.98)^{*}$ \\
\hline Radiotherapy (vs no) & $0.98(0.88,1.09)$ & $1.06(0.92,1.23)^{* *}$ \\
\hline \multicolumn{3}{|l|}{ SES } \\
\hline Low & 1 & 1 \\
\hline Medium & $0.78(0.68,0.88)^{* *}$ & $0.77(0.65,0.91)^{* *}$ \\
\hline High & $0.75(0.66,0.86)^{* *}$ & $0.84(0.70,1.00)^{*}$ \\
\hline Institutionalised & $1.35(1.09,1.68)^{* *}$ & $1.32(0.98,1.78)$ \\
\hline Hypertension (vs no) & $1.12(1.00,1.27)$ & $1.00(0.85,1.20)$ \\
\hline CVD (vs no) & $1.23(1.09,1.40)^{* *}$ & $1.00(0.84,1.20)$ \\
\hline CVA (vs no) & $1.05(0.81,1.34)^{* *}$ & $0.95(0.66,1.36)$ \\
\hline Previous cancer (vs no) & $1.19(1.02,1.38)^{*}$ & $1.00(0.80,1.25)$ \\
\hline Lung disease (vs no) & $1.41(1.20,1.66)^{* *}$ & $1.37(1.10,1.71)^{*}$ \\
\hline
\end{tabular}

Values are HR $(95 \% \mathrm{CI})$

$* p<0.05$

$* * p<0.01$

significantly higher for rectal cancer patients with diabetes than for those without diabetes (HR 1.30, 95\% CI 1.06, 1.41; Table 5). Again, age and sex appeared to be strong confounding variables, changing the HR by more than $10 \%$, whereas addition of having had a previous cancer or other comorbidities to the multivariate model did not significantly change the observed HR. There was no interaction between diabetes and age, stage, number of lymph nodes examined, receipt of adjuvant therapy, SES and year of diagnosis on (disease-specific) mortality among colon or rectal cancer patients.

\section{Discussion}

This population-based study on more than 10,000 stage I-III colorectal cancer patients reveals that diabetes at the time of rectal cancer diagnosis was associated with an HR of 1.30 for the risk of colorectal cancer mortality compared with those without diabetes. Colon cancer patients with diabetes did not have an increased risk of death from colorectal cancer compared with those without. Furthermore, our results confirm previous findings that overall mortality was increased among patients with pre-existing diabetes at colon or rectal cancer diagnosis.

A previous meta-analysis showed that patients diagnosed with cancer who had pre-existing diabetes were at increased risk of long-term, all-cause mortality compared with those without diabetes [7]. It is, however, possible that the excess mortality risk related to diabetes is completely independent of cancer and cancer treatment. In order to understand whether diabetes influences cancer prognosis above and beyond the prognosis by each disease state independently, one should evaluate disease-specific mortality and adjust for potential differences in cancer stage or treatment between patients with and without diabetes. However, the subsidiary meta-analysis of studies that distinguished between cancer and non-cancer mortality was inconclusive [7], as only a few studies have evaluated disease-specific mortality among cancer patients with diabetes. These studies yielded conflicting results because of a small number of diabetes patients, a limited number of deaths, or no information about cancer treatment [12, 13, 15-18]. Nevertheless, recent analyses of a large prospective cohort showed increased allcause mortality, colorectal cancer-specific mortality and CVD-specific mortality among colorectal cancer patients with diabetes [14].

In our study we again observed that colon cancer patients with diabetes less often received adjuvant chemotherapy than non-diabetic cancer patients [5, 25]. This apparently also confounded the association between diabetes and cancer mortality among colon cancer patients. This observation is confirmed in a systematic review that evaluated 34 studies on the impact of comorbidity on chemotherapy use and outcomes [26]. Most studies in this review reported lower use of chemotherapy and worse outcomes among cancer patients with comorbidities [27, 28].

For rectal cancer, we did not observe a different stage distribution or less aggressive treatment in patients with concurrent diabetes. Therefore our current findings may imply that the increased disease-specific mortality risk in rectal cancer patients with diabetes follows from a specific interaction between diabetes and cancer.

Several explanations for a worse disease-specific outcome have been discussed in previous papers. It is possible that diabetic patients respond differently to adjuvant therapies. A small retrospective review in rectal cancer patients showed that diabetic patients $(n=17)$ showed higher local progression rates after neoadjuvant chemoradiotherapy than non-diabetic patients $(n=93)$, whereas none of the diabetic 
patients achieved a pathological complete response [29]. Also, Meyerhardt and colleagues found a significantly higher rate of cancer recurrence and overall mortality among diabetic cancer patients in an adjuvant chemotherapy trial for colon cancer patients, suggesting a smaller effect of therapy [30]. In a review on colorectal cancer outcomes in patients with and without diabetes, an increased risk of cancer recurrence, non-response to chemoradiotherapy and treatment-related complications was reported [8]. However, the authors' overall conclusion was that much of the long-term mortality risk in colorectal cancer patients with diabetes can be attributed to causes other than cancer.

In our study, we did not observe a significant interaction effect between adjuvant chemotherapy or radiotherapy and diabetes on survival. Unfortunately, as in most studies in this field [26], we did not have information about tolerability or toxicity of treatment in diabetes patients.

Another explanation for the increased mortality risk is increased tumour cell proliferation and metastases in a physiological environment of hyperinsulinaemia and hyperglycaemia, leading to a worse prognosis [31]. Studies among non-diabetic breast and colon cancer patients revealed that high levels of fasting insulin had an adverse prognostic effect on distant recurrence and death $[32,33]$. In addition, retrospective, observational studies on diabetic treatment suggest that long-acting insulin glargine (A21Gly, B31Arg,B32Arg human insulin) may increase the risk of cancer [34], whereas biguanide metformin may decrease cancer risk [35, 36] and cancer mortality [37]. Furthermore, obesity and physical inactivity are also associated with disease progression and mortality in non-diabetic colorectal cancer patients [38-43]. The mediators for the elevated risk of colorectal cancer, cancer recurrence and death are not known, but are thought to be related to hyperinsulinaemia, insulin resistance, insulin-like growth factor, adipocytokines and inflammatory cytokines [44, 45]. All these findings suggest that hyperinsulinaemia may stimulate cancer progression, due to its mitogenic effect [46], and support the idea of biological interaction. However, the clinical relevance of the pro-cancer effect of insulin in diabetic patients is still unclear [46].

As we unfortunately did not have information about the diabetes duration and insulin requirement, as well as metabolic control and body mass index, we hope that our planned studies in this field will answer these questions.

Another limitation of this study is the fact that we did not have information about the number of chemotherapy or radiotherapy courses and possible interruption of treatment due to complications in those with or without diabetes. Furthermore, although we believe that a large populationbased study that combines cancer stage and treatment variables with cause-specific mortality data is very much needed in this field, the latter also has limitations. Attribution of cause of death is often problematic, especially for colorectal cancer, where records for the UCOD do not accurately specify between colon and rectal cancers [47]. We observed that $2 \%$ of patients diagnosed with colon cancer had rectal cancer as the UCOD, whereas $34 \%$ of rectal cancer patients had colon cancer as the UCOD. These findings are comparable to a recent US study in which the UCOD records disagreed with California Cancer Registry records for 700 $(6 \%)$ of 11,404 colon cancer deaths and with $1,958(39 \%)$ of 5,011 rectal cancer deaths, and $82 \%$ of the misclassified rectal cancer deaths were coded as colon cancer deaths in the UCOD [47]. Reclassification decreased cause-specific survival for both colon and rectal cancers, but the impact was more pronounced for rectal cancer. Interchangeable use of the terms colon cancer and colorectal cancer is probably one of the reasons for UCOD misclassification. Nevertheless, we believe that by including colon and rectal cancer as the cause of death for both sites, we did not overestimate cause-specific survival. Also, this apparent misclassification is unlikely to have been systematically different for patients with or without diabetes.

Another limitation is the fact that we analysed colorectal cancer death in the presence of competing risks, such as death due to cardiovascular events. As diabetic cancer patients have a higher risk of dying from a cardiovascular event than cancer patients without diabetes, Kaplan-Meier estimates as well as standard Cox regression may have overestimated the actual incidence of cancer death [48]. Therefore it is important not to ignore other causes of death and also include overall mortality analyses to obtain a comprehensive picture of the impact of diabetes on outcomes.

Furthermore, we were not able to include mortality information from the background population without cancer. While most studies among non-cancer populations find standardised mortality ratios of $2-3$ for diabetes vs nondiabetes in a similar age-group [49, 50], this study finds much lower HRs for comparisons of diabetes with non-diabetes.

In conclusion, this large population-based study has confirmed previous findings that overall mortality is increased among patients with pre-existing diabetes at diagnosis of colon or rectal cancer. Most interesting was our observation that diabetes at the time of rectal cancer diagnosis was associated with an increased risk of colorectal cancer mortality compared with no diabetes. As this was not explained by differences in stage or treatment of rectal cancer patients with or without diabetes, there might be a specific interaction between diabetes and rectal cancer. We aim to elucidate pathways in future in-depth studies including detailed diabetes- and cancer-related variables. 
Acknowledgements We thank the following hospitals for their cooperation: Amphia Hospital, Breda; Catharina Hospital, Eindhoven; Elkerliek Hospital, Helmond and Deurne; Jeroen Bosch Hospital, 's Hertogenbosch; Maxima Medical Centre, Eindhoven and Veldhoven; Sint Anna Hospital, Geldrop; St Elisabeth Hospital, Tilburg; Twee Steden Hospital, Tilburg and Waalwijk; VieCury Medical Centre, Venlo and Venray; Instituut Verbeeten, Tilburg.

Funding This study was financed by a grant from the European Federation for the Study of Diabetes (EFSD); L.V. van de PollFranse is supported by a Cancer Research Award from the Dutch Cancer Society (no. UVT-2009-4349).

Duality of interest The authors declare that there is no duality of interest associated with this manuscript.

Contribution statement LP, HH, JWC and VL were responsible for the conception and design of the study, LP, MLG and VL analysed data, all authors interpreted data, LP drafted the article, and HH, JWC, MLG and VL critically reviewed the article. All authors approved the final version submitted for publication.

Open Access This article is distributed under the terms of the Creative Commons Attribution License which permits any use, distribution, and reproduction in any medium, provided the original author(s) and the source are credited.

\section{References}

1. Janssen-Heijnen ML, Houterman S, Lemmens VE, Louwman MW, Maas HA, Coebergh JW (2005) Prognostic impact of increasing age and co-morbidity in cancer patients: a populationbased approach. Crit Rev Oncol Hematol 55:231-240

2. Evans JM, Barnett KN, Ogston SA, Morris AD (2007) Increasing prevalence of type 2 diabetes in a Scottish population: effect of increasing incidence or decreasing mortality? Diabetologia 50:729-732

3. Stovring H, Andersen M, Beck-Nielsen H, Green A, Vach W (2003) Rising prevalence of diabetes: evidence from a Danish pharmaco-epidemiological database. Lancet 362:537-538

4. National Institutes of Health (2008) National Institute of Diabetes and Digestive and Kidney Diseases National Diabetes Statistics, 2007 fact sheet. US Department of Health and Human Services, Bethesda, MD

5. van de Poll-Franse LV, Houterman S, Janssen-Heijnen ML, Dercksen MW, Coebergh JW, Haak HR (2007) Less aggressive treatment and worse overall survival in cancer patients with diabetes: a large population based analysis. Int J Cancer 120:1986-1992

6. Cancer in the Netherlands (2004) Trends, prognoses and implications for healthcare. Signalling Committee Cancer of the Dutch Cancer Society, Amsterdam. ISBN 90-71229-13-0

7. Barone BB, Yeh HC, Snyder CF et al (2008) Long-term all-cause mortality in cancer patients with preexisting diabetes mellitus: a systematic review and meta-analysis. JAMA 300:2754-2764

8. Stein KB, Snyder CF, Barone BB et al (2010) Colorectal cancer outcomes, recurrence, and complications in persons with and without diabetes mellitus: a systematic review and meta-analysis. Dig Dis Sci 55:1839-1851

9. Giovannucci E, Harlan DM, Archer MC et al (2010) Diabetes and cancer: a consensus report. Diabetes Care 33:1674-1685

10. Renehan A, Smith U, Kirkman MS (2010) Linking diabetes and cancer: a consensus on complexity. Lancet 375:2201-2202

11. Siddiqui AA, Spechler SJ, Huerta S, Dredar S, Little BB, Cryer B (2008) Elevated $\mathrm{HbA} 1 \mathrm{c}$ is an independent predictor of aggressive clinical behavior in patients with colorectal cancer: a case-control study. Dig Dis Sci 53:2486-2494

12. Hammarsten J, Hogstedt B (2005) Hyperinsulinaemia: a prospective risk factor for lethal clinical prostate cancer. Eur J Cancer 41:2887-2895

13. Folsom AR, Anderson KE, Sweeney C, Jacobs DR Jr (2004) Diabetes as a risk factor for death following endometrial cancer. Gynecol Oncol 94:740-745

14. Dehal AN, Newton CC, Jacobs EJ, Patel AV, Gapstur SM, Campbell PT (2010) Impact of diabetes mellitus and insulin use on survival after colorectal cancer diagnosis: the Cancer Prevention Study-II Nutrition Cohort. J Clin Oncol 30:53-59

15. Chia VM, Newcomb PA, Trentham-Dietz A, Hampton JM (2007) Obesity, diabetes, and other factors in relation to survival after endometrial cancer diagnosis. Int J Gynecol Cancer 17:441-446

16. Polednak AP (2006) Comorbid diabetes mellitus and risk of death after diagnosis of colorectal cancer: a population-based study. Cancer Detect Prev 30:466-472

17. Will JC, Galuska DA, Vinicor F, Calle EE (1998) Colorectal cancer: another complication of diabetes mellitus? Am J Epidemiol $147: 816-825$

18. Jullumstro E, Kollind M, Lydersen S, Edna TH (2009) Diabetes mellitus and outcomes of colorectal cancer. Acta Oncol 48:361-367

19. Giovannucci E, Harlan DM, Archer MC et al (2010) Diabetes and cancer: a consensus report. CA Cancer J Clin 60:207-221

20. Schouten LJ, Hoppener P, van den Brandt PA, Knottnerus JA, Jager JJ (1993) Completeness of cancer registration in Limburg, the Netherlands. Int J Epidemiol 22:369-376

21. Kieszak SM, Flanders WD, Kosinski AS, Shipp CC, Karp H (1999) A comparison of the Charlson comorbidity index derived from medical record data and administrative billing data. J Clin Epidemiol 52:137-142

22. Charlson ME, Pompei P, Ales KL, MacKenzie CR (1987) A new method of classifying prognostic comorbidity in longitudinal studies: development and validation. J Chronic Dis 40:373-383

23. van Duijn C, Keij I (2002) Sociaal-economische status indicator op postcode niveau. Maandstatistiek van de Bevolking 50:32-35

24. Babyak MA (2004) What you see may not be what you get: a brief, nontechnical introduction to overfitting in regression-type models. Psychosom Med 66:411-421

25. Lemmens VE, van Halteren AH, Janssen-Heijnen ML, Vreugdenhil G, Repelaer van Driel OJ, Coebergh JW (2005) Adjuvant treatment for elderly patients with stage III colon cancer in the southern Netherlands is affected by socioeconomic status, gender, and comorbidity. Ann Oncol 16:767-772

26. Lee L, Cheung WY, Atkinson E, Krzyzanowska MK (2011) Impact of comorbidity on chemotherapy use and outcomes in solid tumors: a systematic review. J Clin Oncol 29:106-117

27. De Marco MF, Janssen-Heijnen ML, van der Heijden LH, Coebergh JW (2000) Comorbidity and colorectal cancer according to subsite and stage: a population-based study. Eur J Cancer 36:95-99

28. Lemmens VE, Janssen-Heijnen ML, Houterman S et al (2007) Which comorbid conditions predict complications after surgery for colorectal cancer? World J Surg 31:192-199

29. Caudle AS, Kim HJ, Tepper JE et al (2008) Diabetes mellitus affects response to neoadjuvant chemoradiotherapy in the management of rectal cancer. Ann Surg Oncol 15:1931-1936

30. Meyerhardt JA, Catalano PJ, Haller DG et al (2003) Impact of diabetes mellitus on outcomes in patients with colon cancer. J Clin Oncol 21:433-440

31. Richardson LC, Pollack LA (2005) Therapy insight: influence of type 2 diabetes on the development, treatment and outcomes of cancer. Nat Clin Pract Oncol 2:48-53

32. Goodwin PJ, Ennis M, Pritchard KI et al (2002) Fasting insulin and outcome in early-stage breast cancer: results of a prospective cohort study. J Clin Oncol 20:42-51 
33. Wolpin BM, Meyerhardt JA, Chan AT et al (2009) Insulin, the insulin-like growth factor axis, and mortality in patients with nonmetastatic colorectal cancer. J Clin Oncol 27:176-185

34. Hemkens LG, Grouven U, Bender R et al (2009) Risk of malignancies in patients with diabetes treated with human insulin or insulin analogues: a cohort study. Diabetologia 52:1732-1744

35. Currie CJ, Poole CD, Gale EA (2009) The influence of glucoselowering therapies on cancer risk in type 2 diabetes. Diabetologia 52:1766-1777

36. Libby G, Donnelly LA, Donnan PT, Alessi DR, Morris AD, Evans JM (2009) New users of metformin are at low risk of incident cancer: a cohort study among people with type 2 diabetes. Diabetes Care 32:1620-1625

37. Landman GW, Kleefstra N, van Hateren KJ, Groenier KH, Gans RO, Bilo HJ (2010) Metformin associated with lower cancer mortality in type 2 diabetes (ZODIAC-16). Diabetes Care 33:322-6

38. Meyerhardt JA, Heseltine D, Niedzwiecki D et al (2006) Impact of physical activity on cancer recurrence and survival in patients with stage III colon cancer: findings from CALGB 89803. J Clin Oncol 24:3535-3541

39. Meyerhardt JA, Giovannucci EL, Holmes MD et al (2006) Physical activity and survival after colorectal cancer diagnosis. J Clin Oncol 24:3527-3534

40. Meyerhardt JA, Catalano PJ, Haller DG et al (2003) Influence of body mass index on outcomes and treatment-related toxicity in patients with colon carcinoma. Cancer 98:484-495
41. Meyerhardt JA, Tepper JE, Niedzwiecki D et al (2004) Impact of body mass index on outcomes and treatment-related toxicity in patients with stage II and III rectal cancer: findings from Intergroup Trial 0114. J Clin Oncol 22:648-657

42. Dignam JJ, Polite BN, Yothers G et al (2006) Body mass index and outcomes in patients who receive adjuvant chemotherapy for colon cancer. J Natl Cancer Inst 98:1647-1654

43. Haydon AM, Macinnis RJ, English DR, Giles GG (2006) Effect of physical activity and body size on survival after diagnosis with colorectal cancer. Gut 55:62-67

44. Calle EE, Kaaks R (2004) Overweight, obesity and cancer: epidemiological evidence and proposed mechanisms. Nat Rev Cancer 4:579-591

45. Sandhu MS, Dunger DB, Giovannucci EL (2002) Insulin, insulinlike growth factor-I (IGF-I), IGF binding proteins, their biologic interactions, and colorectal cancer. J Natl Cancer Inst 94:972-980

46. Vigneri P, Frasca F, Sciacca L, Pandini G, Vigneri R (2009) Diabetes and cancer. Endocr Relat Cancer 16:1103-1123

47. Yin D, Morris CR, Bates JH, German RR (2011) Effect of misclassified underlying cause of death on survival estimates of colon and rectal cancer. J Natl Cancer Inst 103:1130-1133

48. Wolbers M, Koller MT, Witteman JC, Steyerberg EW (2009) Prognostic models with competing risks: methods and application to coronary risk prediction. Epidemiology 20:555-561

49. Carstensen B, Kristensen JK, Ottosen P, Borch-Johnsen K (2008) The Danish National Diabetes Register: trends in incidence, prevalence and mortality. Diabetologia 51:2187-2196

50. Hansen MB, Jensen ML, Carstensen B (2012) Causes of death among diabetic patients in Denmark. Diabetologia 55:294-302 